\title{
On the supercritical KdV equation with time-oscillating nonlinearity
}

\author{
M. Panthee and M. Scialom
}

Abstract. For the initial value problem (IVP) associated to the generalized Korteweg-de Vries (gKdV) equation with supercritical nonlinearity,

$$
u_{t}+\partial_{x}^{3} u+\partial_{x}\left(u^{k+1}\right)=0, \quad k \geq 5,
$$

numerical evidence [3] shows that, there are initial data $\phi \in H^{1}(\mathbb{R})$ such that the corresponding solution may blow-up in finite time. Also, with the evidence from numerical simulation $[1,18]$, it has been claimed that a periodic time dependent coefficient in the nonlinearity would disturb the blow-up solution, either accelerating or delaying it. In this work, we investigate the IVP associated to the gKdV equation

$$
u_{t}+\partial_{x}^{3} u+g(\omega t) \partial_{x}\left(u^{k+1}\right)=0,
$$

where $g$ is a periodic function and $k \geq 5$ is an integer. We prove that, for given initial data $\phi \in H^{1}(\mathbb{R})$, as $|\omega| \rightarrow \infty$, the solution $u_{\omega}$ converges to the solution $U$ of the initial value problem associated to

$$
U_{t}+\partial_{x}^{3} U+m(g) \partial_{x}\left(U^{k+1}\right)=0,
$$

with the same initial data, where $m(g)$ is the average of the periodic function $g$. Moreover, if the solution $U$ is global and satisfies $\|U\|_{L_{x}^{5} L_{t}^{10}}<\infty$, then we prove that the solution $u_{\omega}$ is also global provided $|\omega|$ is sufficiently large.

Mathematics Subject Classification (2000). 35Q35, 35Q53.

Keywords. Korteweg-de Vries equation, Cauchy problem, Local and global well-posedness.

\footnotetext{
M. P. was partially supported by the Research Center of Mathematics of the University of Minho, Portugal through the FCT Pluriannual Funding Program, and through the project PTDC/MAT/109844/2009, and M. S. was partially supported by FAPESP Brazil.
} 


\section{Introduction}

Motivated from an earlier work in [5] for the critical KdV equation, we consider the initial value problem (IVP)

$$
\left\{\begin{array}{l}
u_{t}+\partial_{x}^{3} u+g(\omega t) \partial_{x}\left(u^{k+1}\right)=0, \\
u\left(x, t_{0}\right)=\phi(x),
\end{array}\right.
$$

where $x, t, t_{0}, \omega \in \mathbb{R}$ and $u=u(x, t)$ is a real valued function, $k \geq 5$ is an integer and $g \in C(\mathbb{R}, \mathbb{R})$ is a periodic function with period $L>0$. To make the analysis simple, we translate the initial time $t_{0}$ to 0 and consider the following IVP

$$
\left\{\begin{array}{l}
u_{t}+\partial_{x}^{3} u+g\left(\omega\left(t+t_{0}\right)\right) \partial_{x}\left(u^{k+1}\right)=0, \\
u(x, 0)=\phi(x)
\end{array}\right.
$$

Before analyzing the IVP (1.1) with time oscillating nonlinearity, we discuss some aspects of the supercritical Korteweg-de Vries (KdV) equation,

$$
\begin{cases}u_{t}+\partial_{x}^{3} u+\partial_{x}\left(u^{k+1}\right)=0, & k \geq 5, \\ u(x, 0)=\phi(x), & x, t \in \mathbb{R} .\end{cases}
$$

For $k=4$ the IVP (1.3) is called critical in the literature for three different reasons, see $[4,14]$ and references therein. As described in [14], the first reason is that, for $k=1,2,3$ the solution exists globally for all data in $H^{1}(\mathbb{R})$, while for $k=4$ the global existence holds only for small data (i.e., data with small $H^{1}(\mathbb{R})$-norm). Second reason is that the index $k=4$ is critical for the orbital stability of the solitary wave solutions, see [4]. More precisely, using the arguments from Grillakis et al. [9], Bona et al. [4] prove that the solitary wave solutions of the gKdV equation (1.3) are $H^{1}$-stable if and only if $k<4$ and instable if $k>4$. However, this argument does not apply for the case $k=4$, see also [19]. Finally, the third reason is that the case $k=4$ is the only power for which a solitary wave solution cannot have arbitrarily small $L^{2}$-norm, see [14]. In the light of this observation, the equation (1.3) is known as the supercritical $\mathrm{KdV}$ equation in the literature.

Well-posedness issues for the IVP (1.3) have been extensively studied in the literature, see for example $[10,14,15]$ and references therein. A detailed account of the recent well-posedness results can be found in Kenig et al. [14], where they proved that, there exists $\delta_{k}>0$ such that the IVP (1.3) is globally well-posed for any data $\phi \in H^{s}(\mathbb{R}), s \geq s_{k}:=\frac{1}{2}-\frac{k}{2}$ satisfying $\left\|D_{x}^{s_{k}} \phi\right\|_{L_{x}^{2}}<\delta_{k}$. They were also able to relax the smallness condition on the given data to obtain local well-posedness result, but paying price that the existence time now depends on the shape of the data $\phi$ as well, and not just on its size. These are the best well-posedness results in the sense that $s=s_{k}$ is the critical exponent given by the scaling argument. However, for data in $H^{s}(\mathbb{R}), s>s_{k}$, they were able to remove the size and shape restriction and got local-well posedness for arbitrary data with life span $T$ of the solution depending on $\|\phi\|_{H^{s}(\mathbb{R})}$. Quite recently, Farah et al. [8] considered the IVP (1.3) to address the global well-posedness for the data with low Sobolev regularity. In this context, they 
proved the following local well-posedness result in the function space slightly different from the one used in [14].

Theorem 1.1. [8] Let $k>4$ and $s>s_{k}:=\frac{1}{2}-\frac{k}{2}$. Then for any $\phi \in H^{s}(\mathbb{R})$ there exist $T=T\left(\|\phi\|_{H^{s}(\mathbb{R})}\right)>0$ (with $T(s, \rho) \rightarrow \infty$ as $\rho \rightarrow 0$ ) and a unique strong solution $u$ to the IVP (1.3) satisfying:

$$
\begin{aligned}
& u \in C\left([0, T] ; H^{s}(\mathbb{R})\right), \\
& \left\|\partial_{x} u\right\|_{L_{x}^{\infty} L_{T}^{2}}+\left\|D_{x}^{s} \partial_{x} u\right\|_{L_{x}^{\infty} L_{T}^{2}}<\infty, \\
& \|u\|_{L_{x}^{5} L_{T}^{10}}+\left\|D_{x}^{s} u\right\|_{L_{x}^{5} L_{T}^{10}}<\infty \\
& \left\|D_{t}^{\gamma_{k}} D_{x}^{\alpha_{k}} D_{t}^{\beta_{k}} u\right\|_{L_{x}^{p_{k}} L_{T}^{q_{k}}}<\infty
\end{aligned}
$$

where

$$
\begin{aligned}
& \alpha_{k}=\frac{1}{10}-25 k, \quad \beta_{k}=\frac{3}{10}-\frac{6}{5 k}, \quad \gamma_{k}=\gamma_{k}(s)=\frac{s-s_{k}}{3} \\
& \frac{1}{p_{k}}=\frac{2}{5 k}+\frac{1}{10}, \quad \frac{1}{q_{k}}=\frac{3}{10}-\frac{4}{5 k} .
\end{aligned}
$$

Moreover, for any $T^{\prime} \in(0, T)$, there exists a neighborhood $\mathcal{V}$ of $\phi$ in $H^{s}(\mathbb{R})$ such that the map $\tilde{\phi} \mapsto \tilde{u}$ from $\mathcal{V}$ into the class defined by (1.4) to (1.7) with $T^{\prime}$ in place of $T$ is Lipschitz.

In what follows, we will modify the statement of this result to suit in our context for given data in $H^{1}(\mathbb{R})$ (see Theorem 1.2 below).

We recall that, the $L_{x}^{2}(\mathbb{R})$ norm and energy are conserved by the flow of (1.3). More precisely,

$$
\int_{\mathbb{R}}|u(x, t)|^{2} d x=\int_{\mathbb{R}}|\phi(x)|^{2} d x
$$

and

$$
E(u(\cdot, t)):=\frac{1}{2} \int_{\mathbb{R}}\left\{\left(u_{x}(x, t)\right)^{2}-c_{k} u^{k+2}(x, t)\right\} d x=E(\phi),
$$

are time independent quantities.

The conserved quantities (1.10) and (1.11) yield an a priori estimate for $\left\|\partial_{x} u(t)\right\|_{L^{2}(\mathbb{R})}$ if the initial data $\phi$ is sufficiently small in $H^{1}(\mathbb{R})$. For a detailed work-out of this fact we refer readers to [8]. This a priori estimate allows one to iterate the local solution to get the global one for small data in $H^{1}(\mathbb{R})$. Recently, a numerical study carried out by Bona et al. [3] (see also $[2,4])$ reveled the existence of $H^{1}$-data for which the corresponding solution to the supercritical $\mathrm{KdV}$ equation may blow-up in finite time. This is the point that motivated us to carry on this work in the light of the recent work of Abdullaev et al. [1] and Konotop and Pacciani [18]. In the case of the critical $\mathrm{KdV}$ equation $(k=4)$, there is an extensive series of works carried out by Martel and Merle [19-21] about the finite time blow-up solutions and their stability/instability analysis. For the most resent work in this series, see [22]. As far as we know, for the supercritical case such analytical study does not exist. 
The authors in $[1,18]$ investigate the effect of a time oscillating coefficient in the nonlinearity of the Bose-Einstein condensates. An investigation of solutions which are global for large frequencies is carried out in [1], while in [18], a study of solutions which blow-up in finite time is done. Their results are numerical. Roughly speaking, they claim that the periodic time dependent coefficient in the nonlinearity would disturb the blow-up solution, either by accelerating or delaying it. Recently, Cazenave and Scialom [6] considered the nonlinear Schrödinger (NLS) equation and got an analytical insight to understand the problem by showing that the solution really depends on the frequency of the oscillating term. They proved that the solution $u$ to the IVP associated to the NLS equation

$$
i u_{t}+\Delta u+\theta(\omega t)|u|^{\alpha} u=0, \quad x \in \mathbb{R}^{N},
$$

where $0<\alpha<\frac{4}{(N-2)^{+}}$is an $H^{1}$ sub-critical exponent and $\theta$ is a periodic function, with initial data $\phi \in H^{1}\left(\mathbb{R}^{N}\right)$ converges as $|\omega| \rightarrow \infty$ to the solution $U$ of the limiting equation

$$
i U_{t}+\Delta U+I(\theta)|U|^{\alpha} U=0, \quad x \in \mathbb{R}^{N},
$$

with the same initial data, where $I(\theta)$ is the average of $\theta$. Moreover, they also showed that, if the limiting solution $U$ is global and has a certain decay property as $t \rightarrow \infty$, then $u$ is also global if $|\omega|$ is sufficiently large. A similar result has been proved for the critical KdV equation in our earlier work [5]. In this work, we are interested in addressing the supercritical KdV equation in the same spirit. The numerical evidences for the existence of blow-up solution to $(1.3)$ in $H^{1}(\mathbb{R})$ due to Bona et al. [3] (see also $[2,4]$ ) and the discussion made above strengthen our motivation of studying (1.1) with time oscillating nonlinearity.

As discussed above, our interest here is to investigate the behavior of the solution for given data in $H^{1}(\mathbb{R})$ to the IVP $(1.1)$ as $|\omega| \rightarrow \infty$. The natural limiting candidate to think of is the solution to the following IVP

$$
\begin{cases}U_{t}+\partial_{x}^{3} U+m(g) \partial_{x}\left(U^{k+1}\right)=0, & k \geq 5, \\ U(x, 0)=\phi(x), & x, t \in \mathbb{R},\end{cases}
$$

where $m(g):=\frac{1}{L} \int_{0}^{L} g(t) d t$ is the mean value of $g$ and is a real number. To this end, we need an appropriate well-posedness result for the supercritical KdV equation in $H^{1}(\mathbb{R})$. We recall the local well-posedness result from [8] for arbitrary data in $H^{s}(\mathbb{R}), s>s_{k}$, stated in Theorem 1.1 (See also [14]). The function space used in Theorem 1.1 has an additional norm $\left\|D_{t}^{\gamma_{k}} D_{x}^{\alpha_{k}} D_{t}^{\beta_{k}} u\right\|_{L_{x}^{p_{k}} L_{T}^{q_{k}}}$ that involves time derivatives of the solution. The presence of this norm creates an extra difficulty to handle the time-oscillating nonlinearity. Therefore, to deal with our case, we need to avoid the presence of the norm that involves time derivatives. Also, it is very important to have an explicit expression that provides the local existence time of the solution. In the literature, we did not find an explicitly written proof of the $H^{1}(\mathbb{R})$ well-posedness for the IVP (1.3) that fulfills our requirement. Therefore, we will provide a new proof for the well-posedness of the IVP $(1.3)$ in $H^{1}(\mathbb{R})$. Our proof allows us to extend the 
well-posedness result to the IVP (1.2) and as a consequence to have an estimate of the local existence time.

Other than the recent works $[5,6]$, there are very less works in the literature that address the well-posedness issues for the equations of the $\mathrm{KdV}$ and NLS family with time dependent nonlinearity (see [7,23,24]). The authors in [7] deal with the NLS equation in $\mathbb{R}^{2}$ with nonlinearity of the form $\cos ^{2}(\Omega t)|u|^{p-1} u$ in the critical and supercritical cases. The author in [23] considered the transitional $\mathrm{KdV}$ equation with nonlinearity of the form $f(t) u \partial_{x} u$, where $f$ is a continuous function such that $f^{\prime} \in L_{\text {loc }}^{1}(\mathbb{R})$, and proved the global well-posedness in $H^{s}(\mathbb{R}), s \geq 1$. The transitional $\mathrm{KdV}$ arises in the study of long solitary waves propagating on the thermocline separating two layers of fluids of almost equal densities in which the effect of the change in the depth of the bottom layer, which the wave feels as it approaches the shore, results in the coefficient of the nonlinear term, for details see [17]. In [24], transitional Benjamin-Ono equation with time dependent coefficient in the nonlinearity has been considered and the main result is the global existence of the solution for data in $H^{s}(\mathbb{R}), s \geq \frac{3}{2}$.

Before stating the main results of this work, we define notations that will be used throughout this work.

Notation: We use $\hat{f}$ to denote the Fourier transform of $f$ and is defined as,

$$
\hat{f}(\xi)=\frac{1}{(2 \pi)^{1 / 2}} \int_{\mathbb{R}} e^{-i x \xi} f(x) d x .
$$

The $L^{2}$-based Sobolev space of order $s$ will be denoted by $H^{s}$ with norm

$$
\|f\|_{H^{s}(\mathbb{R})}=\left(\int_{\mathbb{R}}\left(1+\xi^{2}\right)^{s}|\hat{f}(\xi)|^{2} d \xi\right)^{1 / 2} .
$$

The Riesz potential of order $-s$ is denoted by $D_{x}^{s}=\left(-\partial_{x}^{2}\right)^{s / 2}$. For $f$ : $\mathbb{R} \times[0, T] \rightarrow \mathbb{R}$ we define the mixed $L_{x}^{p} L_{T}^{q}$-norm by

$$
\|f\|_{L_{x}^{p} L_{T}^{q}}=\left(\int_{\mathbb{R}}\left(\int_{0}^{T}|f(x, t)|^{q} d t\right)^{p / q} d x\right)^{1 / p}
$$

with usual modifications when $p=\infty$. We replace $T$ by $t$ if $[0, T]$ is the whole real line $\mathbb{R}$. We use the notation $f \in H^{\alpha+}$ if $f \in H^{\alpha+\epsilon}$ for $\epsilon>0$.

We define two more spaces $X_{T}$ and $Y_{T}$ with norms

$$
\begin{aligned}
\|f\|_{X_{T}}:= & \|f\|_{L_{T}^{\infty} H^{1}}+\left\|\partial_{x} f\right\|_{L_{x}^{\infty} L_{T}^{2}}+\left\|\partial_{x}^{2} f\right\|_{L_{x}^{\infty} L_{T}^{2}} \\
& +\|f\|_{L_{x}^{5} L_{T}^{10}}+\left\|\partial_{x} f\right\|_{L_{x}^{5} L_{T}^{10}}+\left\|\partial_{x} f\right\|_{L_{x}^{20} L_{T}^{5 / 2}}+\|f\|_{L_{x}^{4} L_{T}^{\infty}}
\end{aligned}
$$

and

$$
\|f\|_{Y_{T}}:=\left\|\partial_{x} f\right\|_{L_{x}^{2} L_{T}^{2}}+\|f\|_{L_{x}^{2} L_{T}^{2}},
$$

respectively. We replace $X_{T}$ by $X_{t}$ or $X_{(T, \infty)}$, if the time integral is taken in the interval $(0, \infty)$ or $(T, \infty)$ respectively, and similarly for $Y_{T}$. 
We use the letter $C$ to denote various constants whose exact values are immaterial and which may vary from one line to the next.

First, let us state the $H^{1}$-local well-posedness result for the IVP (1.3) in a function space that does not use norms involving time derivatives of the solution.

Theorem 1.2. Suppose $\phi \in H^{1}(\mathbb{R})$. Then there exist $T=T\left(\|\phi\|_{H^{1}(\mathbb{R})}\right)>0$ and a unique solution $u$ to the IVP (1.3) satisfying

$$
\begin{aligned}
& u \in C\left([0, T] ; H^{1}(\mathbb{R})\right), \\
& \left\|\partial_{x} u\right\|_{L_{x}^{\infty} L_{T}^{2}}+\left\|\partial_{x}^{2} u\right\|_{L_{x}^{\infty} L_{T}^{2}}<\infty, \\
& \|u\|_{L_{x}^{5} L_{T}^{10}}+\left\|\partial_{x} u\right\|_{L_{x}^{5} L_{T}^{10}}+\left\|\partial_{x} u\right\|_{L_{x}^{20} L_{T}^{5 / 2}}<\infty, \\
& \|u\|_{L_{x}^{4} L_{T}^{\infty}}<\infty .
\end{aligned}
$$

Moreover, for any $T^{\prime} \in(0, T)$, there exists a neighborhood $\mathcal{V}$ of $\phi$ in $H^{1}(\mathbb{R})$ such that the map $\tilde{\phi} \mapsto \tilde{u}$ from $\mathcal{V}$ into the class defined by (1.17) to (1.20) with $T^{\prime}$ in place of $T$ is Lipschitz.

Using Duhamel's principle, we prove Theorem 1.2 by considering the integral equation associated to the $\operatorname{IVP}(1.3)$,

$$
u(t)=S(t) \phi-\int_{0}^{t} S\left(t-t^{\prime}\right) \partial_{x}\left(u^{k+1}\right)\left(t^{\prime}\right) d t^{\prime},
$$

where $S(t)$ is the unitary group generated by the operator $\partial_{x}^{3}$ that describes the solution to the linear problem. Our interest is to solve (1.21) using the contraction mapping principle in an appropriate metric space.

Remark 1.3. Since the average $m(g)$ of $g$ is a constant, the proof of Theorem 1.2 can be adapted line by line to obtain a similar well-posedness result for the IVP (1.14). The only difference in this case is that, to complete the contraction argument we need to choose $T>0$ in such a way that $C|m(g)| T^{1 / 2}\|\phi\|_{H^{1}(\mathbb{R})}^{k}<$ $\frac{1}{2}$. So the existence time $T$ depends on $|m(g)|$ and $\|\phi\|_{H^{1}(\mathbb{R})}$. We also have the following bound

$$
\|U\|_{X_{T}} \leq C\|\phi\|_{H^{1}(\mathbb{R})}, \quad \forall t \in[0, T] .
$$

Regarding the well-posedness results for the IVP (1.2), we have the following theorem.

Theorem 1.4. Suppose $\phi \in H^{1}(\mathbb{R})$. Then there exist $T=T\left(\|\phi\|_{H^{1}(\mathbb{R})},\|g\|_{L^{\infty}}\right)>$ 0 and $a$ unique solution $u_{\omega, t_{0}} \in C\left([0, T] ; H^{1}(\mathbb{R})\right)$ to the IVP (1.2) satisfying (1.18)-(1.20)

Moreover, for any $T^{\prime} \in(0, T)$, there exists a neighborhood $\mathcal{V}$ of $\phi$ in $H^{1}(\mathbb{R})$ such that the map $\tilde{\phi} \mapsto \tilde{u}_{\omega, t_{0}}$ from $\mathcal{V}$ into the class defined by (1.17) to (1.20) with $T^{\prime}$ in place of $T$ is Lipschitz.

Now, we state the main results of this work.

Theorem 1.5. Fix $\phi \in H^{1}(\mathbb{R})$. For given $\omega, t_{0} \in \mathbb{R}$, let $u_{\omega, t_{0}}$ be the maximal solution of the IVP (1.2) and $U$ be the solution of the limiting IVP (1.14) 
defined on the maximal time of existence $\left[0, S_{\max }\right)$. Then, for given any $0<$ $T<S_{\max }$, the solution $u_{\omega, t_{0}}$ exists on $[0, T]$ for all $t_{0} \in \mathbb{R}$ and $|\omega|$ large. Moreover, $\left\|u_{\omega, t_{0}}-U\right\|_{X_{T}} \rightarrow 0$, as $|\omega| \rightarrow \infty$, uniformly in $t_{0} \in \mathbb{R}$. In particular, the convergence holds in $C\left([0, T] ; H^{1}(\mathbb{R})\right)$ for all $T \in\left(0, S_{\max }\right)$.

Theorem 1.6. Let $\phi \in H^{1}(\mathbb{R})$ and $u_{\omega, t_{0}}$ be the maximal solution of the IVP (1.1). Suppose U be the maximal solution of the IVP (1.14) defined on $\left[0, S_{\max }\right)$. If $S_{\max }=\infty$ and

$$
\|U\|_{L_{x}^{5} L_{t}^{10}}<\infty,
$$

then it follows that $u_{\omega, t_{0}}$ is global for all $t_{0} \in \mathbb{R}$ if $|w|$ is sufficiently large. Moreover,

$$
\left\|u_{\omega, t_{0}}-U\right\|_{X_{t}} \rightarrow 0, \text { when }|w| \rightarrow \infty,
$$

uniformly in $t_{0}$. In particular, convergence holds in $L^{\infty}\left((0, \infty) ; H^{1}(\mathbb{R})\right)$.

In view of the numerical prediction in [3] of the existence of blow-up solution for the supercritical $\mathrm{KdV}$ equation in $H^{1}(\mathbb{R})$, the Theorem 1.6 is very interesting in the sense that when $m(g) \leq 0$ and $k$ is even the solution $U$ to the IVP (1.14) will be global for all $H^{1}$-data (see [8]) and the solution $u_{\omega, t_{0}}$ to the nonlinear problem (1.2) will be global too, for $|\omega|$ large enough.

Before leaving this section, we discuss the example constructed in [6] in the context of the NLS equation with time oscillating nonlinearity. The authors in [6] showed that for small frequency $|\omega|$, the solution $u_{\omega, t_{0}}$ blows-up in finite time or is global depending on $t_{0}$, while for the large frequency $|\omega|$, the solution $u_{\omega, t_{0}}$ is global for all $t_{0} \in \mathbb{R}$. The same example can be utilized with small modification in the context of the supercritical $\mathrm{KdV}$ equation. We present it here for the convenience of the readers.

Example 1.7. Let $L>1,0<\epsilon<\frac{L-1}{2}$ and consider a periodic function $g$ defined by

$$
m(g)=0, \quad \text { and } \quad g(s)=\left\{\begin{array}{l}
1,|s| \leq \epsilon \\
0,1 \leq s \leq 1+\epsilon
\end{array}\right.
$$

with period $L$.

Fix $\phi \in H^{1}(\mathbb{R})$ and assume that the solution $v$ of the IVP

$$
\left\{\begin{array}{l}
v_{t}+v_{x x x}+v^{k+1} \partial_{x} v=0, \quad k \geq 5 \\
v(x, 0)=\phi(x)
\end{array}\right.
$$

blows-up in finite time, say $T^{*}$. In the light of the numerical evidences presented in $[2,3]$ (see also [4]) we can suppose that such a solution $v(x, t)$ of (1.26) with $t \in\left[0, T^{*}\right)$, exists.

From Theorem 1.5, for this particular $\phi$ and the periodic function $g$, we have that the solution $u_{\omega, t_{0}}$ to the IVP (1.2) converges, as $|\omega| \rightarrow \infty$, to the solution $U$ of the linear $\mathrm{KdV}$ equation with same initial data $\phi$. So, in view of Theorem 1.6, $u_{\omega, t_{0}}$ is global as $|\omega| \rightarrow \infty$ for all $t_{0} \in \mathbb{R}$.

Now we move to analyze the behavior of the solution for $|\omega|$ small. Note that $g(\omega s)=1$ when $|\omega s| \leq \epsilon$. Therefore, if we consider $|\omega|<\frac{\epsilon}{T^{*}}$, then we see that the solution $v$ to the $\operatorname{IVP}(1.26)$ satisfies $(1.2)$ for $t_{0}=0$ on $\left[0, T^{*}\right)$. 
By uniqueness, $u_{\omega, 0}=v$. Hence the solution $u_{\omega, 0}$ of the IVP (1.2) blows-up in finite time, provided $|\omega|<\frac{\epsilon}{T^{*}}$.

Let $\epsilon=\epsilon(A)$ be as in Corollary 3.5 with $A=\|g\|_{L_{t}^{\infty}}$. From the linear estimate (2.6) we have that $S(\cdot) \phi \in L_{x}^{5} L_{t}^{10}$, so there exists $T>0$ such that

$$
\|S(\cdot)[S(T) \phi]\|_{L_{x}^{5} L_{t}^{10}}=\|S(\cdot) \phi\|_{L_{x}^{5} L_{(T, \infty)}^{10}} \leq \epsilon .
$$

For $\omega>0$, if we consider $t_{0}=\frac{1}{\omega}$, we have that $g\left(\omega\left(s+t_{0}\right)\right)=0$ for all $1 \leq \omega\left(s+t_{0}\right) \leq 1+\epsilon$, i.e., for all $0 \leq s \leq \frac{\epsilon}{\omega}$. Therefore, if we let $\omega>0$ satisfying $\omega \leq \frac{\epsilon}{T}$ (i.e., $\left.T \leq \frac{\epsilon}{\omega}\right)$, and choose $t_{0}=\frac{1}{\omega}$, then $g\left(\omega\left(s+t_{0}\right)\right)=0$ for all $0 \leq s \leq T$. So, with this choice, $u_{\omega, t_{0}}$ solves the linear KdV equation if $0 \leq t \leq T$. Therefore, for $\omega \leq \frac{\epsilon}{T}, u_{\omega, t_{0}}$ exists on $[0, T]$ and is given by $S(t) \phi$, in particular $u_{\omega, t_{0}}(T)=S(T) \phi$. From (1.27), $\left\|S(\cdot) u_{\omega, t_{0}}(T)\right\|_{L_{x}^{5} L_{t}^{10}} \leq \epsilon$. Hence, from Corollary 3.5 we conclude that $u_{\omega, t_{0}}$ is global.

This paper is organized as follows. In Sect. 2 we record some preliminary estimates associated to the linear problem and other relevant results. In Sect. 3 we give a proof of the local well-posedness result for the supercritical KdV equation in $H^{1}(\mathbb{R})$ and some other results that will be used in the proof of the main Theorems. Finally, the proof of the main results will be given in Sect. 4.

\section{Preliminary estimates}

In this section we record some linear estimates associated to the IVP (1.1). These estimates are not new and can be found in the literature. For the sake of clearness we sketch the ideas involved and provide references where a detailed proof can be found.

Lemma 2.1. If $u_{0} \in L^{2}(\mathbb{R})$, then

$$
\left\|\partial_{x} S(t) u_{0}\right\|_{L_{x}^{\infty} L_{t}^{2}} \leq C\left\|u_{0}\right\|_{L_{x}^{2}}
$$

If $f \in L_{x}^{1} L_{t}^{2}$, then

$$
\left\|\partial_{x} \int_{0}^{t} S\left(t-t^{\prime}\right) f\left(\cdot, t^{\prime}\right) d t^{\prime}\right\|_{L_{t}^{\infty} L_{x}^{2}} \leq C\|f\|_{L_{x}^{1} L_{t}^{2}},
$$

and

$$
\left\|\partial_{x}^{2} \int_{0}^{t} S\left(t-t^{\prime}\right) f\left(\cdot, t^{\prime}\right) d t^{\prime}\right\|_{L_{x}^{\infty} L_{t}^{2}} \leq C\|f\|_{L_{x}^{1} L_{t}^{2}} .
$$

Proof. For the proof of the homogeneous smoothing effect (2.1) and the double smoothing effect (2.3), see Theorem 3.5 in [14] (see also Section 4 in [13]). The inequality (2.2) is the dual version of (2.1).

Now we give the maximal function estimate.

Lemma 2.2. If $u_{0} \in \dot{H}^{1 / 4}(\mathbb{R})$, then

$$
\left\|S(t) u_{0}\right\|_{L_{x}^{4} L_{T}^{\infty}} \leq C\left\|D_{x}^{1 / 4} u_{0}\right\|_{L^{2}(\mathbb{R})} .
$$


Also, we have

$$
\left\|S(t) u_{0}\right\|_{L_{x}^{\infty} L_{T}^{\infty}} \leq C\left\|u_{0}\right\|_{H^{\frac{1}{2}+}(\mathbb{R})} .
$$

Proof. For the proof of the estimate (2.4) we refer to Theorem 3.7 in [14] (see also [12] and [16]). The estimate (2.5) follows from Sobolev embedding. analysis.

In what follows, we state some more estimates that will be used in our

Lemma 2.3. If $u_{0} \in L^{2}(\mathbb{R})$, then

$$
\left\|S(t) u_{0}\right\|_{L_{x}^{5} L_{t}^{10}} \leq C\left\|u_{0}\right\|_{L_{x}^{2}}
$$

Also we have

$$
\left\|\partial_{x} S(t) u_{0}\right\|_{L_{x}^{20} L_{t}^{5 / 2}} \leq C\left\|D_{x}^{1 / 4} u_{0}\right\|_{L_{x}^{2}} .
$$

Proof. The proof of the estimates (2.6) and (2.7) can be found in Corollary 3.8 and Proposition 3.17 in [14] respectively.

Lemma 2.4. Let $u_{0} \in L_{x}^{2}$, then for any $(\theta, \alpha) \in[0,1] \times\left[0, \frac{1}{2}\right]$, we have

$$
\left\|D_{x}^{\theta \alpha / 2} S(t) u_{0}\right\|_{L_{T}^{q} L_{x}^{p}} \leq C\left\|u_{0}\right\|_{L_{x}^{2}}
$$

where $(q, p)=\left(\frac{6}{\theta(\alpha+1)}, \frac{2}{1-\theta}\right)$.

Proof. See Lemma 2.4 in [11].

We state next the Leibniz's rule for fractional derivatives whose proof is given in [14], Theorem A.8.

Lemma 2.5. Let $\alpha \in(0,1), \alpha_{1}, \alpha_{2} \in[0, \alpha], \alpha_{1}+\alpha_{2}=\alpha$. Let $p, p_{1}, p_{2}, q, q_{1}, q_{2} \in$ $(1, \infty)$ be such that $\frac{1}{p}=\frac{1}{p_{1}}+\frac{1}{p_{2}}, \frac{1}{q}=\frac{1}{q_{1}}+\frac{1}{q_{2}}$. Then

$$
\left\|D_{x}^{\alpha}(f g)-f D_{x}^{\alpha} g-g D_{x}^{\alpha} f\right\|_{L_{x}^{p} L_{T}^{q}} \leq C\left\|D_{x}^{\alpha_{1}} f\right\|_{L_{x}^{p_{1}} L_{T}^{q_{1}}}\left\|D_{x}^{\alpha_{2}} g\right\|_{L_{x}^{p_{2}} L_{T}^{q_{2}}} .
$$

Moreover, for $\alpha_{1}=0$ the value $q_{1}=\infty$ is allowed.

Definition 2.6. Let $1 \leq p, q \leq \infty,-\frac{1}{4} \leq \alpha \leq 1$. We say that a triple $(p, q, \alpha)$ is an admissible triple if

$$
\frac{1}{p}+\frac{1}{2 q}=\frac{1}{4} \quad \text { and } \quad \alpha=\frac{2}{q}-\frac{1}{p} .
$$

Proposition 2.7. For any admissible triples $\left(p_{j}, q_{j}, \alpha_{j}\right), j=1,2$, the following estimate holds

$$
\left\|D_{x}^{\alpha_{1}} \int_{0}^{t} S\left(t-t^{\prime}\right) f\left(\cdot, t^{\prime}\right) d t^{\prime}\right\|_{L_{x}^{p_{1}} L_{t}^{q_{1}}} \leq C\left\|D_{x}^{-\alpha_{2}} f\right\|_{L_{x}^{p_{2}^{\prime}} L_{t}^{q_{2}^{\prime}}},
$$

where $p_{2}^{\prime}, q_{2}^{\prime}$ are the conjugate exponents of $p_{2}, q_{2}$.

Proof. For the proof we refer to Proposition 2.3 in [15].

The following results will be used to complete the contraction mapping argument. 
Lemma 2.8. Let $X_{T}$ and $Y_{T}$ be the spaces defined earlier and $S$ be the unitary group associated to the operator $\partial_{x}^{3}$, then we have

$$
\begin{gathered}
\left\|S(t) u_{0}\right\|_{X_{T}} \leq C_{0}\left\|u_{0}\right\|_{H^{1}(\mathbb{R})}, \\
\left\|\int_{0}^{t} S\left(t-t^{\prime}\right) f\left(t^{\prime}\right) d t^{\prime}\right\|_{X_{T}} \leq C T^{1 / 2}\|f\|_{Y_{T}} .
\end{gathered}
$$

Proof. The estimate (2.12) follows from the linear estimates in Lemmas 2.1, 2.2 and 2.3. For the proof of the estimate (2.13), we refer to our earlier work in $[5]$.

Lemma 2.9. The following estimate holds,

$$
\left\|\partial_{x}\left(u^{k+1}\right)\right\|_{Y_{T}} \leq C\|u\|_{X_{T}}^{k+1} .
$$

Proof. The idea of the proof is similar to the one we used in [5] for the critical $\mathrm{KdV}$ equation. Using Hölder's inequality and the fact that $H^{1}(\mathbb{R}) \hookrightarrow L^{\infty}(\mathbb{R})$, we get

$$
\begin{aligned}
\left\|\partial_{x}\left(u^{k+1}\right)\right\|_{L_{x}^{2} L_{T}^{2}} & \leq C\left\|u^{k-2}\right\|_{L_{x}^{\infty} L_{T}^{\infty}}\left\|u^{2} \partial_{x} u\right\|_{L_{x}^{2} L_{T}^{2}} \\
& \leq C\|u\|_{L_{T}^{\infty} H^{1}(\mathbb{R})}^{k-2}\|u\|_{L_{x}^{4} L_{T}^{\infty}}^{2}\left\|\partial_{x} u\right\|_{L_{x}^{\infty} L_{T}^{2}} .
\end{aligned}
$$

Similarly

$$
\begin{aligned}
\left\|\partial_{x}^{2}\left(u^{k+1}\right)\right\|_{L_{x}^{2} L_{T}^{2}} \leq C\left[\left\|u^{k-1}\left(\partial_{x} u\right)^{2}\right\|_{L_{x}^{2} L_{T}^{2}}+\left\|u^{k} \partial_{x}^{2} u\right\|_{L_{x}^{2} L_{T}^{2}}\right] \\
\leq C\left[\left\|u^{k-2}\right\|_{L_{x}^{\infty} L_{T}^{\infty}}\left\|u\left(\partial_{x} u\right)^{2}\right\|_{L_{x}^{2} L_{T}^{2}}+\left\|u^{k-2}\right\|_{L_{x}^{\infty} L_{T}^{\infty}}\left\|u^{2} \partial_{x}^{2} u\right\|_{L_{x}^{2} L_{T}^{2}}\right] \\
\leq C\|u\|_{L_{T}^{\infty} H^{1}(\mathbb{R})}^{k-2}\left[\|u\|_{L_{x}^{4} L_{T}^{\infty}}\left\|\partial_{x} u\right\|_{L_{x}^{5} L_{T}^{10}}\left\|\partial_{x} u\right\|_{L_{x}^{20} L_{T}^{5 / 2}}\right. \\
\left.\quad+\|u\|_{L_{x}^{4} L_{T}^{\infty}}^{k-2}\left\|\partial_{x}^{2} u\right\|_{L_{x}^{\infty} L_{T}^{2}}\right] .
\end{aligned}
$$

In view of definitions of $X_{T}$-norm and $Y_{T}$-norm, the estimates $(2.15)$ and (2.16) yield the required result (2.14).

The following result from [6] will also be useful in our analysis.

Lemma 2.10. Let $T>0,1 \leq p<q \leq \infty$ and $A, B \geq 0$. If $f \in L^{q}(0, T)$ satisfies

$$
\|f\|_{L_{(0, t)}^{q}} \leq A+B\|f\|_{L_{(0, t)}^{p}}
$$

for all $t \in(0, T)$, then there exists a constant $K=K(B, p, q, T)$ such that

$$
\|f\|_{L_{(0, T)}^{q}} \leq K A .
$$

\section{Proof of the well-posedness results}

We start this section by proving the well-posedness results for the IVP (1.3) announced in Theorem 1.2. 
Proof of Theorem 1.2. For $a>0$, consider a ball in $X_{T}$ defined by

$$
\mathcal{B}_{a}^{T}=\left\{u \in C\left([0, T]: X_{T}(\mathbb{R})\right):\|u\|_{X_{T}}<a\right\} .
$$

Our aim is to show that, there exist $a>0$ and $T>0$, such that the application $\Phi$ defined by

$$
\Phi(u):=S(t) \phi-\int_{0}^{t} S\left(t-t^{\prime}\right) \partial_{x}\left(u^{k+1}\right)\left(t^{\prime}\right) d t^{\prime},
$$

maps $\mathcal{B}_{a}^{T}$ into $\mathcal{B}_{a}^{T}$ and is a contraction.

Using the estimates (2.13) and (2.14), we obtain

$$
\begin{aligned}
\|\Phi\|_{X_{T}} & \leq C_{0}\|\phi\|_{H^{1}}+C T^{1 / 2}\left\|\partial_{x}\left(u^{k+1}\right)\right\|_{Y_{T}} \\
& \leq C_{0}\|\phi\|_{H^{1}}+C T^{1 / 2}\|u\|_{X_{T}}^{k+1} .
\end{aligned}
$$

Hence, for $u \in \mathcal{B}_{a}^{T}$,

$$
\|\Phi\|_{X_{T}} \leq C_{0}\|\phi\|_{H^{1}}+C T^{1 / 2} a^{k+1} .
$$

Now, choose $a=2 C_{0}\|\phi\|_{H^{1}}$ and $T$ such that $C T^{1 / 2} a^{k}<1 / 2$. With these choices we get, from (3.3),

$$
\|\Phi\|_{X_{T}} \leq \frac{a}{2}+\frac{a}{2} .
$$

Therefore, $\Phi$ maps $\mathcal{B}_{a}^{T}$ into $\mathcal{B}_{a}^{T}$.

With the similar argument, one can prove that $\Phi$ is a contraction. The rest of the proof follows standard argument.

Remark 3.1. From the choice of $a$ and $T$ in the proof of Theorem 1.2 it is clear that the local existence time is given by

$$
T \leq C\|\phi\|_{H^{1}(\mathbb{R})}^{-2 k} .
$$

Moreover, we have the following bound,

$$
\|u\|_{X_{T}} \leq C\|\phi\|_{H^{1}(\mathbb{R})} .
$$

In what follows, we sketch a proof for the local well-posedness result for the IVP (1.2).

Proof of Theorem 1.4. As in the proof of Theorem 1.2, this theorem will also be proved by considering the integral equation associated to the IVP (1.2),

$$
u(t)=S(t) \phi-\int_{0}^{t} S\left(t-t^{\prime}\right) g\left(\omega\left(t^{\prime}+t_{0}\right)\right) \partial_{x}\left(u^{k+1}\right)\left(t^{\prime}\right) d t^{\prime},
$$

and using the contraction mapping principle.

First of all, notice that the periodic function $g$ is bounded, say $\|g\|_{L_{t}^{\infty}} \leq$ $A$, for some positive constant $A$. Since the norms involved in the space $Y$ permit us to take out $\|g\|_{L_{t}^{\infty}}$-norm as a coefficient, the proof of this theorem follows exactly the same argument as in the proof of Theorem 1.2. Moreover, as the initial data $\phi$ is the same, the choice of the radius $a$ of the ball is exactly the same. However, to complete the contraction mapping argument, we must 
select $T>0$ such that $C\|g\|_{L_{t}^{\infty}} T^{1 / 2} a^{4}<\frac{1}{2}$, which implies that the existence $T$ is given by

$$
T=T\left(\|g\|_{L_{t}^{\infty}},\|\phi\|_{H^{1}(\mathbb{R})}\right)=\frac{C}{\|g\|_{L_{t}^{\infty}}^{2}\|\phi\|_{H^{1}(\mathbb{R})}^{2 k}} .
$$

Furthermore, in this case too, from the proof, one can get

$$
\|u\|_{X_{T}} \leq C\|\phi\|_{H^{1}(\mathbb{R})} .
$$

In sequel, we present some results that play a central role in the proof of the main theorems of this work. We begin with the following lemma whose proof can be found in [5].

Lemma 3.2. Let $X_{T}$ and $Y_{T}$ be spaces as defined in (1.15) and (1.16). Let $f \in Y_{T}$, then we have the following convergence

$$
\int_{0}^{t} g\left(\omega\left(t^{\prime}+t_{0}\right)\right) S\left(t-t^{\prime}\right) f\left(t^{\prime}\right) d t^{\prime} \rightarrow m(g) \int_{0}^{t} S\left(t-t^{\prime}\right) f\left(t^{\prime}\right) d t^{\prime},
$$

whenever $|\omega| \rightarrow \infty$, in the $X_{T}$-norm

With the similar argument as in the case of the critical KdV equation (see [5]), we have the following convergence result.

Lemma 3.3. Let the initial data $\phi \in H^{1}(\mathbb{R})$. Let $u_{\omega, t_{0}}$ be the maximal solution of the IVP (1.1). Suppose $U$ be the maximal solution of the IVP (1.14) defined in $\left[0, S_{\max }\right)$. Let $0<T<S_{\max }$ and let $u_{\omega, t_{0}}$ exists in $[0, T]$ for $|\omega|$ large and that

$$
\limsup _{|\omega| \rightarrow \infty} \sup _{t_{0} \in \mathbb{R}}\left\|u_{\omega, t_{0}}\right\|_{L_{T}^{\infty} H^{1}(\mathbb{R})}<\infty
$$

and

$$
\limsup _{|\omega| \rightarrow \infty} \sup _{t_{0} \in \mathbb{R}}\left\|u_{\omega, t_{0}}\right\|_{L_{x}^{4} L_{T}^{\infty}}<\infty .
$$

Then, for all $t \in[0, T]$,

$$
\sup _{t_{0} \in \mathbb{R}}\left\|u_{\omega, t_{0}}-U\right\|_{X_{T}} \rightarrow 0, \quad \text { as }|\omega| \rightarrow \infty .
$$

In particular, $u_{\omega, t_{0}} \rightarrow U$ as $|\omega| \rightarrow \infty$, in $H^{1}(\mathbb{R})$.

Proof. Since $u_{\omega, t_{0}}$ and $U$ have the same initial data $\phi$, from Duhamel's formula, we have

$$
\begin{aligned}
u_{\omega, t_{0}}-U= & \int_{0}^{t} g\left(\omega\left(t^{\prime}+t_{0}\right)\right) S\left(t-t^{\prime}\right) \partial_{x}\left(u_{\omega, t_{0}}^{k+1}\right) d t^{\prime}-m(g) \int_{0}^{t} S\left(t-t^{\prime}\right) \partial_{x}\left(U^{k+1}\right) d t^{\prime} \\
= & \int_{0}^{t} g\left(\omega\left(t^{\prime}+t_{0}\right)\right) S\left(t-t^{\prime}\right) \partial_{x}\left(u_{\omega, t_{0}}^{k+1}-U^{k+1}\right) d t^{\prime} \\
& +\int_{0}^{t}\left[g\left(\omega\left(t^{\prime}+t_{0}\right)\right)-m(g)\right] S\left(t-t^{\prime}\right) \partial_{x}\left(U^{k+1}\right) d t^{\prime} \\
= & : I_{1}+I_{2} .
\end{aligned}
$$


We note that

$$
\left|u^{k+1}-v^{k+1}\right| \leq C\left(|u|^{k}+|v|^{k}\right)|u-v|
$$

and

$$
\begin{aligned}
\left|\partial_{x}\left(u^{k+1}-v^{k+1}\right)\right| \leq & C\left[\left(|u|^{k}+|v|^{k}\right)\left|\partial_{x}(u-v)\right|\right. \\
& \left.+\left(\left|\partial_{x} u\right|+\left|\partial_{x} v\right|\right)\left(|u|^{k-1}+|v|^{k-1}\right)|u-v|\right] .
\end{aligned}
$$

Let $\|g\|_{L_{T}^{\infty}} \leq A$. Use of $(2.2)$, (3.14), Hölder's inequality and the assumptions (3.10) and (3.11), yield

$$
\begin{aligned}
\left\|I_{1}\right\|_{L_{T}^{\infty} L_{x}^{2}} & \leq C\|g\|_{L_{T}^{\infty}}\left\|u_{\omega, t_{0}}^{k+1}-U^{k+1}\right\|_{L_{x}^{1} L_{T}^{2}} \\
& \leq C A\left\|u_{\omega, t_{0}}^{k}\left(u_{\omega, t_{0}}-U\right)\right\|_{L_{x}^{1} L_{T}^{2}}+\left\|U^{k}\left(u_{\omega, t_{0}}-U\right)\right\|_{L_{x}^{1} L_{T}^{2}} \\
& \leq C A\left\|u_{\omega, t_{0}}^{k}\right\|_{L_{x}^{2} L_{T}^{\infty}}\left\|u_{\omega, t_{0}}-U\right\|_{L_{x}^{2} L_{T}^{2}}+\left\|U^{k}\right\|_{L_{x}^{2} L_{T}^{\infty}}\left\|u_{\omega, t_{0}}-U\right\|_{L_{x}^{2} L_{T}^{2}} \\
& \leq C A\left[\left\|u_{\omega, t_{0}}^{k-2}\right\|_{L_{x}^{\infty} L_{T}^{\infty}}\left\|u_{\omega, t_{0}}^{2}\right\|_{L_{x}^{2} L_{T}^{\infty}}+\left\|U^{k-2}\right\|_{L_{x}^{\infty} L_{T}^{\infty}}\left\|U^{2}\right\|_{L_{x}^{2} L_{T}^{\infty}}\right]\left\|u_{\omega, t_{0}}-U\right\|_{L_{T}^{2} L_{x}^{2}} \\
& \leq C A\left[\left\|u_{\omega, t_{0}}\right\|_{L_{T}^{\infty} H^{1}(\mathbb{R})}^{k-2}\left\|u_{\omega, t_{0}}\right\|_{L_{x}^{4} L_{T}^{\infty}}^{2}+\|U\|_{L_{T}^{\infty} H^{1}(\mathbb{R})}^{k-2}\|U\|_{L_{x}^{4} L_{T}^{\infty}}^{2}\right]\left\|u_{\omega, t_{0}}-U\right\|_{L_{T}^{2} L_{x}^{2}} \\
& \leq C A\left\|u_{\omega, t_{0}}-U\right\|_{L_{T}^{2} L_{x}^{2}} .
\end{aligned}
$$

Again, using (2.2) and (3.15), one can obtain

$$
\begin{aligned}
\left\|\partial_{x} I_{1}\right\|_{L_{T}^{\infty} L_{x}^{2} \leq} & C A\left\|\partial_{x}\left(u_{\omega, t_{0}}^{k+1}-U^{k+1}\right)\right\|_{L_{x}^{1} L_{T}^{2}} \\
\leq & C A\left[\left\|\left(\left|u_{\omega, t_{0}}\right|^{k}+|U|^{k}\right) \partial_{x}\left(u_{\omega, t_{0}}-U\right)\right\|_{L_{x}^{1} L_{T}^{2}}\right. \\
& \left.+\left\|\left(\left|\partial_{x} u_{\omega, t_{0}}\right|+\left|\partial_{x} U\right|\right)\left(\left|u_{\omega, t_{0}}\right|^{k-1}+|U|^{k-1}\right)\left(u_{\omega, t_{0}}-U\right)\right\|_{L_{x}^{1} L_{T}^{2}}\right] \\
= & : C A\left[J_{1}+J_{2}\right] .
\end{aligned}
$$

With the same argument as in (3.16)

$$
J_{1} \leq C\left\|\partial_{x}\left(u_{\omega, t_{0}}-U\right)\right\|_{L_{T}^{2} L_{x}^{2}}
$$

Now we move to estimate the first term, $\left\|u_{\omega, t_{0}}^{k-1} \partial_{x} u_{\omega, t_{0}}\left(u_{\omega, t_{0}}-U\right)\right\|_{L_{x}^{1} L_{T}^{2}}$ in $J_{2}$, the estimates for the other terms are similar. We have,

$$
\begin{aligned}
& \left\|u_{\omega, t_{0}}^{k-1} \partial_{x} u_{\omega, t_{0}}\left(u_{\omega, t_{0}}-U\right)\right\|_{L_{x}^{1} L_{T}^{2}} \\
& \quad \leq C\left\|u_{\omega, t_{0}}^{2}\right\|_{L_{x}^{2} L_{T}^{\infty}}\left\|u_{\omega, t_{0}}^{k-3} \partial_{x} u_{\omega, t_{0}}\left(u_{\omega, t_{0}}-U\right)\right\|_{L_{x}^{2} L_{T}^{2}} \\
& \quad \leq C\left\|u_{\omega, t_{0}}\right\|_{L_{x}^{4} L_{T}^{\infty}}^{2}\left\|u_{\omega, t_{0}}^{k-3}\right\|_{L_{T}^{\infty} L_{x}^{\infty}}\left\|\partial_{x} u_{\omega, t_{0}}\right\|_{L_{T}^{\infty} L_{x}^{2}}\left\|\left(u_{\omega, t_{0}}-U\right)\right\|_{L_{T}^{2} L_{x}^{\infty}} \\
& \quad \leq C\left\|u_{\omega, t_{0}}\right\|_{L_{x}^{4} L_{T}^{\infty}}^{2}\left\|u_{\omega, t_{0}}\right\|_{L_{T}^{\infty} H^{1}(\mathbb{R})}^{k-2}\left\|\left(u_{\omega, t_{0}}-U\right)\right\|_{L_{T}^{2} H^{1}(\mathbb{R})} \\
& \quad \leq C\left\|\left(u_{\omega, t_{0}}-U\right)\right\|_{L_{T}^{2} H^{1}(\mathbb{R})} .
\end{aligned}
$$

Inserting (3.18) and (3.19) in (3.17), we get

$$
\left\|\partial_{x} I_{1}\right\|_{L_{T}^{\infty} L_{x}^{2}} \leq C A\left\|\left(u_{\omega, t_{0}}-U\right)\right\|_{L_{T}^{2} H^{1}(\mathbb{R})} .
$$

Combining (3.16) and (3.20), we obtain

$$
\left\|I_{1}\right\|_{L_{T}^{\infty} H^{1}(\mathbb{R})} \leq C A\left\|\left(u_{\omega, t_{0}}-U\right)\right\|_{L_{T}^{2} H^{1}(\mathbb{R})} .
$$

From Lemma 3.2, we have

$$
\left\|I_{2}\right\|_{L_{T}^{\infty} H^{1}(\mathbb{R})} \leq C_{\omega} \rightarrow 0, \quad \text { as }|\omega| \rightarrow \infty .
$$


Therefore, we have

$$
\left\|u_{\omega, t_{0}}-U\right\|_{L_{T}^{\infty} H^{1}(\mathbb{R})} \leq C A\left\|\left(u_{\omega, t_{0}}-U\right)\right\|_{L_{T}^{2} H^{1}(\mathbb{R})}+C_{\omega} .
$$

Applying Lemma 2.10 in (3.23), we get

$$
\left\|u_{\omega, t_{0}}-U\right\|_{L_{T}^{\infty} H^{1}(\mathbb{R})} \leq K C_{\omega} \rightarrow 0, \quad \text { as }|\omega| \rightarrow \infty .
$$

From (3.23) and (3.24), it is easy to conclude that

$$
\left\|\left(u_{\omega, t_{0}}-U\right)\right\|_{L_{T}^{2} H^{1}(\mathbb{R})} \rightarrow 0, \quad \text { as }|\omega| \rightarrow \infty .
$$

Now, we move to estimate the other norms involved in the definition of $X_{T}$. Let,

$$
\begin{aligned}
\mathfrak{L}_{1}:= & \left\|\partial_{x}\left(u_{\omega, t_{0}}-U\right)\right\|_{L_{x}^{\infty} L_{T}^{2}}+\left\|\partial_{x}^{2}\left(u_{\omega, t_{0}}-U\right)\right\|_{L_{x}^{\infty} L_{T}^{2}} \\
& +\left\|u_{\omega, t_{0}}-U\right\|_{L_{x}^{5} L_{T}^{10}}+\left\|D_{x}\left(u_{\omega, t_{0}}-U\right)\right\|_{L_{x}^{5} L_{T}^{10}}
\end{aligned}
$$

and

$$
\mathfrak{L}_{2}:=\left\|\partial_{x}\left(u_{\omega, t_{0}}-U\right)\right\|_{L_{x}^{20} L_{T}^{5 / 2}}+\left\|u_{\omega, t_{0}}-U\right\|_{L_{x}^{4} L_{T}^{\infty}} .
$$

Use of $(2.2),(2.3)$, the estimate (2.11) from Proposition 2.7 with admissible triples $\left(p_{1}, q_{1}, \alpha_{1}\right)=(5,10,0)$, and $\left(p_{2}, q_{2}, \alpha_{2}\right)=(\infty, 2,1)$ in $(3.13)$, yields

$$
\mathfrak{L}_{\mathbf{1}} \leq C A\left\|\partial_{x}\left(u_{\omega, t_{0}}^{k+1}-U^{k+1}\right)\right\|_{L_{x}^{1} L_{T}^{2}}+C A\left\|u_{\omega, t_{0}}^{k+1}-U^{k+1}\right\|_{L_{x}^{1} L_{T}^{2}}+\left\|I_{2}\right\|_{X_{T}} .
$$

Therefore, with the same argument as in (3.16)-(3.20), we can obtain

$$
\mathfrak{L}_{\mathbf{I}} \leq C A\left\|u_{\omega, t_{0}}-U\right\|_{L_{T}^{2} H^{1}}+C_{\omega} .
$$

Hence, using Lemma 3.2 and (3.25) we get from (3.27) that

$$
\mathfrak{L}_{1} \stackrel{|\omega| \rightarrow \infty}{\rightarrow} 0 .
$$

Finally, to estimate $\mathfrak{L}_{2}$ we use Proposition 2.7 with admissible triples $\left(p_{1}, q_{1}, \alpha_{1}\right)=(20,5 / 2,3 / 4)$ and $\left(p_{2}, q_{2}, \alpha_{2}\right)=(20 / 3,5,1 / 4)$, to get

$$
\left\|\partial_{x} \int_{0}^{t} S\left(t-t^{\prime}\right) f\left(\cdot, t^{\prime}\right) d t^{\prime}\right\|_{L_{x}^{20} L_{T}^{5 / 2}} \leq C\|f\|_{L_{x}^{20 / 17} L_{T}^{5 / 4}},
$$

and with admissible triples $\left(p_{1}, q_{1}, \alpha_{1}\right)=(4, \infty,-1 / 4)$, and $\left(p_{2}, q_{2}, \alpha_{2}\right)=$ $(20 / 3,5,1 / 4)$, to have

$$
\left\|\int_{0}^{t} S\left(t-t^{\prime}\right) f\left(\cdot, t^{\prime}\right) d t^{\prime}\right\|_{L_{x}^{4} L_{T}^{\infty}} \leq C\|f\|_{L_{x}^{20 / 17} L_{T}^{5 / 4}} .
$$

Using (3.29), (3.30), and the definition of $X_{T}$, we get from (3.13) that

$$
\mathfrak{L}_{2} \leq C A\left\|\partial_{x}\left(u_{\omega, t_{0}}^{k+1}-U^{k+1}\right)\right\|_{L_{x}^{20 / 17} L_{T}^{5 / 4}}+\left\|I_{2}\right\|_{X_{T}}
$$


Using (3.15), we can obtain

$$
\begin{aligned}
\left\|\partial_{x}\left(u_{\omega, t_{0}}^{k+1}-U^{k+1}\right)\right\|_{L_{x}^{20 / 17} L_{T}^{5 / 4} \leq} & C\left[\left\|\left(\left|u_{\omega, t_{0}}\right|^{k}+|U|^{k}\right) \partial_{x}\left(u_{\omega, t_{0}}-U\right)\right\|_{L_{x}^{20 / 17} L_{T}^{5 / 4}}\right. \\
& +\|\left(\left|\partial_{x} u_{\omega, t_{0}}\right|+\left|\partial_{x} U\right|\right)\left(\left|u_{\omega, t_{0}}\right|^{k-1}\right. \\
& \left.\left.+|U|^{k-1}\right)\left(u_{\omega, t_{0}}-U\right) \|_{\left.L_{x}^{20 / 17} L_{T}^{5 / 4}\right]}\right] \\
= & : C\left[\tilde{J}_{1}+\tilde{J}_{2}\right] .
\end{aligned}
$$

Hölder's inequality, the fact that 20/13 > 10/7, Sobolev immersion and the assumption (3.10), imply that

$$
\begin{aligned}
\tilde{J}_{1} & \leq C\left\|\partial_{x}\left(u_{\omega, t_{0}}-U\right)\right\|_{L_{x}^{5} L_{T}^{10}}\left\{\left\|u_{\omega, t_{0}}^{k}\right\|_{L_{x}^{20 / 13} L_{T}^{10 / 7}}+\left\|U^{k}\right\|_{L_{x}^{20 / 13} L_{T}^{10 / 7}}\right\} \\
& \leq C\left\|\partial_{x}\left(u_{\omega, t_{0}}-U\right)\right\|_{L_{x}^{5} L_{T}^{10}}\left\{\left\|u_{\omega, t_{0}}^{k}\right\|_{L_{T}^{10 / 7} L_{x}^{20 / 13}}+\left\|U^{k}\right\|_{L_{T}^{10 / 7} L_{x}^{20 / 13}}\right\} \\
& \leq C\left\|\partial_{x}\left(u_{\omega, t_{0}}-U\right)\right\|_{L_{x}^{5} L_{T}^{10}} T^{7 / 10}\left\{\left\|u_{\omega, t_{0}}\right\|_{L_{T}^{\infty} H^{1}}^{k}+\|U\|_{L_{T}^{\infty} H^{1}}^{k}\right\} \\
& \leq C T^{7 / 10}\left\|\partial_{x}\left(u_{\omega, t_{0}}-U\right)\right\|_{L_{x}^{5} L_{T}^{10}}
\end{aligned}
$$

As in (3.17), we give details in estimating the first term, $\| u_{\omega, t_{0}}^{k-1} \partial_{x} u_{\omega, t_{0}}$ $\left(u_{\omega, t_{0}}-U\right) \|_{L_{x}^{20 / 17} L_{T}^{5 / 4}}$ in $\tilde{J}_{2}$, the estimates for the other terms are similar. Here too, Hölder's inequality, the fact that $20 / 3>5$, Sobolev immersion and the assumption (3.10), yield

$$
\begin{aligned}
& \left\|u_{\omega, t_{0}}^{k-1} \partial_{x} u_{\omega, t_{0}}\left(u_{\omega, t_{0}}-U\right)\right\|_{L_{x}^{20 / 17} L_{T}^{5 / 4}} \\
& \quad \leq C\left\|u_{\omega, t_{0}}^{k-1}\right\|_{L_{x}^{20 / 3} L_{T}^{5}}\left\|\partial_{x} u_{\omega, t_{0}}\right\|_{L_{x}^{2} L_{T}^{2}}\left\|u_{\omega, t_{0}}-U\right\|_{L_{x}^{5} L_{T}^{10}} \\
& \quad \leq C\left\|u_{\omega, t_{0}}^{k-1}\right\|_{L_{T}^{5} L_{x}^{20 / 3}}\left\|\partial_{x} u_{\omega, t_{0}}\right\|_{L_{T}^{2} L_{x}^{2}}\left\|u_{\omega, t_{0}}-U\right\|_{L_{x}^{5} L_{T}^{10}} \\
& \quad \leq C T^{7 / 10}\left\|u_{\omega, t_{0}}\right\|_{L_{T}^{\infty} H^{1}}^{k}\left\|u_{\omega, t_{0}}-U\right\|_{L_{x}^{5} L_{T}^{10}} \\
& \quad \leq C T^{7 / 10}\left\|u_{\omega, t_{0}}-U\right\|_{L_{x}^{5} L_{T}^{10}} .
\end{aligned}
$$

In view of (3.32), (3.33) and (3.34), we get from (3.31) that

$$
\mathfrak{L}_{2} \leq C A T^{7 / 10}\left\{\left\|\partial_{x}\left(u_{\omega, t_{0}}-U\right)\right\|_{L_{x}^{5} L_{T}^{10}}+\left\|u_{\omega, t_{0}}-U\right\|_{L_{x}^{5} L_{T}^{10}}\right\}+C_{\omega} .
$$

Therefore, Lemma 3.2 and (3.28), imply

$$
\mathfrak{L}_{2} \stackrel{|\omega| \rightarrow \infty}{\rightarrow} 0
$$

Now, the proof of the Lemma follows by combining (3.24), (3.28) and (3.36).

In what follows, as we did in our earlier work [5], consider the supercritical KdV equation with more general time dependent coefficient on the nonlinearity

$$
\left\{\begin{array}{l}
u_{t}+u_{x x x}+h(t) \partial_{x}\left(u^{k+1}\right)=0, x, t \in \mathbb{R}, k \geq 5 \\
u(x, 0)=\phi(x)
\end{array}\right.
$$

where $h \in L^{\infty}$ is given. 
The results for the IVP (3.37) and their proofs that we are going to present here are quite similar to the ones we have for the critical KdV equation in [5]. For the sake of clarity, we reproduce them here.

Proposition 3.4. Given any $A>0$, there exist $\epsilon=\epsilon(A)$ and $B>0$ such that if $\|h\|_{L^{\infty}} \leq A$ and if $\phi \in H^{1}(\mathbb{R})$ satisfies

$$
\|S(t) \phi\|_{L_{x}^{5} L_{t}^{10}} \leq \epsilon,
$$

then the corresponding solution $u$ of (3.37) is global and satisfies

$$
\begin{array}{r}
\|u\|_{L_{x}^{5} L_{t}^{10}} \leq 2\|S(t) \phi\|_{L_{x}^{5} L_{t}^{10}}, \\
\|u\|_{X_{t}} \leq B\|\phi\|_{H^{1}(\mathbb{R})} .
\end{array}
$$

Conversely, if the solution $u$ of (3.37) is global and satisfies

$$
\|u\|_{L_{x}^{5} L_{t}^{10}} \leq \epsilon
$$

then

$$
\|S(t) \phi\|_{L_{x}^{5} L_{t}^{10}} \leq 2\|u\|_{L_{x}^{5} L_{t}^{10}} .
$$

Proof. Since $\|h\|_{L_{t}^{\infty}} \leq A$, as in Theorem 1.4 we can prove the local well-posedness for the IVP (3.37) in $H^{1}(\mathbb{R})$ with time of existence $T=$ $T\left(\|\phi\|_{H^{1}(\mathbb{R})},\|h\|_{L^{\infty}}\right)$. Let $u \in C\left(\left[0, T_{\max }\right) ; H^{1}(\mathbb{R})\right)$ be the maximal solution of the IVP (3.37). For $0 \leq t<T_{\max }$, we have that

$$
u(t)=S(t) \phi+w(t),
$$

where

$$
w(t)=-\int_{0}^{t} S\left(t-t^{\prime}\right) h\left(t^{\prime}\right) \partial_{x}\left(u^{k+1}\right)\left(t^{\prime}\right) d t^{\prime} .
$$

Using (2.11) from Proposition 2.7 for admissible triples $(5,10,0)$ and $(\infty, 2,1)$, we obtain

$$
\begin{aligned}
\|w\|_{L_{x}^{5} L_{T}^{10}} & \leq C A\left\|u^{k+1}\right\|_{L_{x}^{1} L_{T}^{2}} \leq C A\left\|u^{k-4}\right\|_{L_{x}^{\infty} L_{T}^{\infty}}\left\|u^{5}\right\|_{L_{x}^{1} L_{T}^{2}} \\
& \leq C A\|u\|_{L_{T}^{\infty} H^{1}}^{k-4}\|u\|_{L_{x}^{5} L_{T}^{10}}^{5} \leq C A\|u\|_{L_{x}^{5} L_{T}^{10}}^{5} .
\end{aligned}
$$

From (3.43) and (3.44) it follows that

$$
\left|\|u\|_{L_{x}^{5} L_{T}^{10}}-\|S(t) \phi\|_{L_{x}^{5} L_{T}^{10}}\right| \leq C A\|u\|_{L_{x}^{5} L_{T}^{10}}^{5} .
$$

Thus, for all $T \in\left(0, T_{\max }\right)$ one has

$$
\|u\|_{L_{x}^{5} L_{T}^{10}} \leq \epsilon+C A\|u\|_{L_{x}^{5} L_{T}^{10}}^{5} .
$$

Choose $\epsilon=\epsilon(A)$ such that

$$
C A(2 \epsilon)^{4}<1 / 2,
$$

and suppose that the estimate (3.38) holds. As the norm is continuous on $T$ and vanishes at $T=0$, using continuity argument, the estimate (3.46) and the choice of $\epsilon$ in (3.47), imply that

$$
\|u\|_{L_{x}^{5} L_{T_{\max }^{10}}} \leq 2 \epsilon .
$$


Moreover, from (3.45)

$$
\begin{aligned}
\|u\|_{L_{x}^{5} L_{T_{\max }}^{10}} & \leq\|S(t) \phi\|_{L_{x}^{5} L_{T_{\max }}^{10}}+C A\|u\|_{L_{x}^{5} L_{T_{\max }^{10}}^{5}} \\
& \leq\|S(t) \phi\|_{L_{x}^{5} L_{T_{\max }^{10}}^{10}}+C A(2 \epsilon)^{4}\|u\|_{L_{x}^{5} L_{T_{\max }}^{10}} .
\end{aligned}
$$

Therefore, with the choice of $\epsilon$ satisfying (3.47), the estimate (3.49) yields

$$
\|u\|_{L_{x}^{5} L_{T_{\max }}^{10}} \leq 2\|S(t) \phi\|_{L_{x}^{5} L_{T_{\max }}^{10}} .
$$

In what follows, we will show that $T_{\max }=\infty$. The inequalities $(2.2),(2.3)$, (2.11) with admissible triples $(5,10,0)$ and $(\infty, 2,1)$, and Hölder's inequality imply

$$
\begin{aligned}
& \|w\|_{L_{T}^{\infty} H^{1}}+\left\|\partial_{x} w\right\|_{L_{x}^{\infty} L_{T}^{2}}+\left\|\partial_{x}^{2} w\right\|_{L_{x}^{\infty} L_{T}^{2}}+\|w\|_{L_{x}^{5} L_{T}^{10}}+\left\|\partial_{x} f\right\|_{L_{x}^{5} L_{T}^{10}} \\
& \quad \leq C A\|u\|_{L_{x}^{5} L_{T}^{10}}^{4}\|u\|_{X_{T}} .
\end{aligned}
$$

Now using (3.29), (3.30) and Hölder's inequality, we have

$$
\begin{aligned}
\left\|\partial_{x} w\right\|_{L_{x}^{20} L_{T}^{5 / 2}}+\|w\|_{L_{x}^{4} L_{T}^{\infty}} & \leq C A\left\|\partial_{x}\left(u^{k+1}\right)\right\|_{L_{x}^{20 / 17} L_{T}^{5 / 4}} \\
& \leq C A\left\|u^{k}\right\|_{L_{x}^{5 / 4} L_{T}^{5 / 2}}\left\|\partial_{x} u\right\|_{L_{x}^{20} L_{T}^{5 / 2}} \\
& \leq C A\left\|u^{k-4}\right\|_{L_{x}^{\infty} L_{T}^{\infty}}\left\|u^{4}\right\|_{L_{x}^{5 / 4} L_{T}^{5 / 2}}\left\|\partial_{x} u\right\|_{L_{x}^{20} L_{T}^{5 / 2}} \\
& \leq C A\|u\|_{L_{T}^{\infty} H^{1}}^{k-4}\|u\|_{L_{x}^{5} L_{T}^{10}}^{4}\left\|\partial_{x} u\right\|_{L_{x}^{20} L_{T}^{5 / 2}} \\
& \leq C A\|u\|_{L_{x}^{5} L_{T}^{10}}^{4}\left\|\partial_{x} u\right\|_{L_{x}^{20} L_{T}^{5 / 2}}
\end{aligned}
$$

Combining (3.51) and (3.52), we obtain

$$
\|w\|_{X_{T}} \leq C A\|u\|_{L_{x}^{5} L_{T}^{10}}^{4}\|u\|_{X_{T}} .
$$

This estimate with (3.47) and (3.48) gives

$$
\|w\|_{X_{T}} \leq C A(2 \epsilon)^{4}\|u\|_{X_{T}}<\frac{1}{2}\|u\|_{X_{T}} .
$$

Using (3.43) we obtain

$$
\|u\|_{X_{T}} \leq\|S(t) \phi\|_{X_{T}}+\|w\|_{X_{T}} \leq C\|\phi\|_{H^{1}(\mathbb{R})}+\frac{1}{2}\|u\|_{X_{T}},
$$

for all $T \in\left(0, T_{\max }\right)$. Therefore, we have

$$
\|u\|_{X_{T_{\max }} \leq 2 C\|\phi\|_{H^{1}(\mathbb{R})} .}
$$

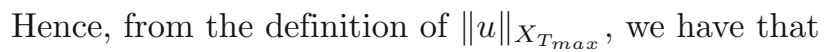

$$
\|u\|_{L_{T_{\max }^{\infty}}^{\infty} H^{1}(\mathbb{R})} \leq C\|u(0)\|_{H^{1}(\mathbb{R})} .
$$

Now, combining the local existence from Theorem 1.4 and the estimate (3.57), the blow-up alternative implies that $T_{\max }=\infty$. Finally, the estimates (3.50) and (3.56) yield (3.39) and (3.40) respectively with $B=2 C$.

Conversely, let $T_{\max }=\infty$ and (3.41) holds. With the similar argument as in (3.45), we can get

$$
\left|\|u\|_{L_{x}^{5} L_{t}^{10}}-\|S(t) \phi\|_{L_{x}^{5} L_{t}^{10}}\right| \leq C A\|u\|_{L_{x}^{5} L_{t}^{10}}^{5} .
$$


Thus, from (3.58) in view of (3.41) and (3.47), one has

$$
\|S(t) \phi\|_{L_{x}^{5} L_{t}^{10}} \leq\|u\|_{L_{x}^{5} L_{t}^{10}}+C A \epsilon^{4}\|u\|_{L_{x}^{5} L_{t}^{10}} \leq 2\|u\|_{L_{x}^{5} L_{t}^{10}} .
$$

Corollary 3.5. Let $h \in L^{\infty}(\mathbb{R})$ satisfy $\|h\|_{L^{\infty}} \leq A$ and $\epsilon$ and $B$ be as in Proposition 3.4. Given $\phi \in H^{1}(\mathbb{R})$, let $u$ be the solution of the IVP (3.37) defined on the maximal interval $\left[0, T_{\max }\right)$. If there exists $T \in\left(0, T_{\max }\right)$ such that

$$
\|S(t) u(T)\|_{L_{x}^{5} L_{t}^{10}} \leq \epsilon,
$$

then the solution $u$ is global. Moreover

$$
\|u\|_{L_{x}^{5} L_{(T, \infty)}^{10}} \leq 2 \epsilon, \quad \text { and } \quad\|u\|_{X_{(T, \infty)}} \leq B\|u(T)\|_{H^{1}(\mathbb{R})} .
$$

Proof. The proof follows by using a standard extension argument. For details we refer to the proof of Corollary 2.4 in [6].

\section{Proof of the main results}

The argument in the proof of the main results, Theorem 1.5 and Theorem 1.6, is quite similar to the one used in the case of the critical $\mathrm{KdV}$ equation [5]. As mentioned earlier, Lemma 3.3 and the local existence Theorem 1.4 are used in the proof of Theorem 1.5. While, Proposition 3.4 and Theorem 1.5 are crucial in the proof of Theorem 1.6. Here we adapt the techniques used in [5] and [6] to complete the proofs.

Proof of Theorem 1.5. Let $A=\|g\|_{L^{\infty}}, T \in\left(0, S_{\max }\right)$ fixed and set

$$
M_{0}=2 \sup _{t \in[0, T]}\|U(t)\|_{H^{1}(\mathbb{R})} .
$$

In particular, for $t=0,(4.1)$ gives $\|\phi\|_{H^{1}(\mathbb{R})} \leq M_{0} / 2$. From Theorem 1.4, we have that for all $\omega, t_{0} \in \mathbb{R}, u_{\omega, t_{0}}$ exists on $[0, \delta]$. Using (3.7) we have that the existence time $\delta$, is given by

$$
\delta=\frac{C}{A^{2} M_{0}^{8}} .
$$

Moreover, from (3.8)

$$
\limsup _{|w| \rightarrow \infty} \sup _{t_{0} \in \mathbb{R}}\left\|u_{\omega, t_{0}}\right\|_{L_{\delta}^{\infty} H^{1}(\mathbb{R})} \leq C\|\phi\|_{H^{1}(\mathbb{R})}
$$

and

$$
\limsup _{|w| \rightarrow \infty} \sup _{t_{0} \in \mathbb{R}}\left\|u_{\omega, t_{0}}\right\|_{L_{x}^{4} L_{\delta}^{\infty} H^{1}(\mathbb{R})} \leq C\|\phi\|_{H^{1}(\mathbb{R})}
$$

From Lemma 3.3, we have that $\sup _{t_{0} \in \mathbb{R}}\left\|u_{\omega, t_{0}}-U\right\|_{X_{T}} \stackrel{|w| \rightarrow \infty}{\longrightarrow} 0$, in particular

$$
\sup _{t_{0} \in \mathbb{R}}\left\|u_{\omega, t_{0}}(\delta)-U(\delta)\right\|_{H^{1}(\mathbb{R})} \stackrel{|w| \rightarrow \infty}{\longrightarrow} 0 .
$$

Combining (4.1) and (4.5), for $|w|$ sufficiently large, we deduce that

$$
\sup _{t_{0} \in \mathbb{R}}\left\|u_{\omega, t_{0}}(\delta)\right\|_{H^{1}(\mathbb{R})} \leq M_{0}
$$


We suppose $\delta \leq T$, otherwise we are done. Using Theorem 1.4 we can extend the solution $u_{\omega, t_{0}}$ (as in the proof of Corollary 3.5) on the interval $[0,2 \delta]$, with $\left\|\tilde{u}_{\omega, t_{0}}\right\|_{L_{t}^{\infty}(0, \delta) H^{1}(\mathbb{R})} \leq C\left\|\tilde{u}_{\omega, t_{0}}(0)\right\|_{H^{1}(\mathbb{R})}$, where $\tilde{u}_{\omega, t_{0}}(t)=u_{\omega, t_{0}}(t+\delta)$ i.e., $\left\|u_{\omega, t_{0}}\right\|_{L_{t}^{\infty}(\delta, 2 \delta) H^{1}(\mathbb{R})} \leq C\left\|u_{\omega, t_{0}}(\delta)\right\|_{H^{1}(\mathbb{R})} \leq C^{2}\|\phi\|_{H^{1}(\mathbb{R})}$. Therefore, (4.3) gives

$$
\limsup _{|w| \rightarrow \infty} \sup _{t_{0} \in \mathbb{R}}\left\|u_{\omega, t_{0}}\right\|_{L_{t}^{\infty}(0,2 \delta) H^{1}(\mathbb{R})} \leq C(1+C)\|\phi\|_{H^{1}(\mathbb{R})} .
$$

Similarly, from (4.4),

$$
\limsup _{|w| \rightarrow \infty} \sup _{t_{0} \in \mathbb{R}}\left\|u_{\omega, t_{0}}\right\|_{L_{x}^{4} L_{2 \delta}^{\infty} H^{1}(\mathbb{R})} \leq C(1+C)\|\phi\|_{H^{1}(\mathbb{R})} .
$$

So, we can again apply the Lemma 3.3. Iterating this argument at a finite number of times with the same time of existence in each iteration, we see that

$$
\limsup _{|w| \rightarrow \infty} \sup _{t_{0} \in \mathbb{R}}\left\|u_{\omega, t_{0}}\right\|_{L_{T}^{\infty} H^{1}(\mathbb{R})} \leq C\|\phi\|_{H^{1}(\mathbb{R})}
$$

and

$$
\limsup _{|w| \rightarrow \infty} \sup _{t_{0} \in \mathbb{R}}\left\|u_{\omega, t_{0}}\right\|_{L_{x}^{4} L_{T}^{\infty}} \leq C\|\phi\|_{H^{1}(\mathbb{R})} .
$$

The result is therefore a consequence of Lemma 3.3.

Proof of Theorem 1.6. Let $\epsilon \in(0, \epsilon(A))$, where $\epsilon(A)$ is as in Proposition 3.4. If $T$ is sufficiently large, from (1.23), we have that

$$
\|U\|_{L_{x}^{5} L_{(T, \infty)}^{10}} \leq \frac{\epsilon}{4} .
$$

Applying Proposition 3.4 to the global solution $\tilde{U}(t)=U(t+T)$, the inequality (3.42) gives

$$
\|S(t) U(T)\|_{L_{x}^{5} L_{t}^{10}}=\|S(t) \tilde{U}(0)\|_{L_{x}^{5} L_{t}^{10}} \leq 2\|\tilde{U}\|_{L_{x}^{5} L_{t}^{10}}=2\|U\|_{L_{x}^{5} L_{(T, \infty)}^{10}} \leq \frac{\epsilon}{2} .
$$

From this inequality and Corollary 3.5 we get

$$
\|U\|_{X_{(T ; \infty)}} \leq B\|U(T)\|_{H^{1}(\mathbb{R})} \text {. }
$$

From Theorem 1.5 it follows that

$$
\sup _{t_{0} \in \mathbb{R}} \sup _{0 \leq t \leq T}\left\|u_{\omega, t_{0}}(t)-U(t)\right\|_{H^{1}(\mathbb{R})} \rightarrow 0, \quad \text { as } \quad|\omega| \rightarrow \infty .
$$

Thus, if $|w|$ is sufficiently large, the triangular inequality along with (4.12) gives

$$
\begin{aligned}
\left\|S(t) u_{\omega, t_{0}}(T)\right\|_{L_{x}^{5} L_{t}^{10}} & \leq\left\|S(t) u_{\omega, t_{0}}(T)-S(t) U(T)\right\|_{L_{x}^{5} L_{t}^{10}}+\|S(t) U(T)\|_{L_{x}^{5} L_{t}^{10}} \\
& \leq\left\|u_{\omega, t_{0}}(T)-U(T)\right\|_{L_{x}^{2}}+\frac{\epsilon}{2} \\
& \leq \epsilon
\end{aligned}
$$

Therefore, Corollary 3.5 implies that $u_{\omega, t_{0}}$ is global. Moreover,

$$
\sup _{t_{0} \in \mathbb{R}}\left\|u_{\omega, t_{0}}\right\|_{L_{x}^{5} L_{(T, \infty)}^{10}} \leq 2 \epsilon, \quad \text { and } \quad\left\|u_{\omega, t_{0}}\right\|_{X_{(T, \infty)}} \leq B\left\|u_{\omega, t_{0}}(T)\right\|_{H^{1}(\mathbb{R})}
$$

for $|w|$ sufficiently large. 
Let $M_{0}=\sup _{0 \leq t \leq T}\|U(t)\|_{H^{1}(\mathbb{R})}$, as in (4.1). Now, we move to prove (1.24). The inequalities (4.12) and (4.14) show that there exists $L>0$ such that

$\sup \sup _{\sup }\left\|u_{\omega, t_{0}}(t)\right\|_{H^{1}(\mathbb{R})} \leq\left(1+M_{0}\right)+B\left\|u_{\omega, t_{0}}(T)\right\|_{H^{1}(\mathbb{R})}=M_{1}<\infty$. $|w| \geq L t_{0} \in \mathbb{R} t \geq 0$

In what follows, we prove that $u_{\omega, t_{0}} \rightarrow U$ in the $\|\cdot\|_{X_{t}}$-norm, when $|\omega| \rightarrow \infty$.

Using Duhamel's formulas for $u_{\omega, t_{0}}$ and $U$ we have

$$
\begin{aligned}
u_{\omega, t_{0}}(T+t)-U(T+t)= & S(t)\left(u_{\omega, t_{0}}(T)-U(T)\right) \\
& -\int_{0}^{t} S\left(t-t^{\prime}\right) g\left(\omega\left(T+t^{\prime}+t_{0}\right)\right) \partial_{x}\left(u_{\omega, t_{0}}^{k+1}\right)\left(T+t^{\prime}\right) d t^{\prime} \\
& +m(g) \int_{0}^{t} S\left(t-t^{\prime}\right) \partial_{x}\left(U^{k+1}\right)\left(T+t^{\prime}\right) d t^{\prime} \\
= & : I_{1}+I_{2}+I_{3} .
\end{aligned}
$$

Using properties of the unitary group $S(t)$ we have by (4.12) that

$$
\left\|I_{1}\right\|_{X_{t}}=\left\|S(t)\left(u_{\omega, t_{0}}(T)-U(T)\right)\right\|_{X_{t}} \leq C\left\|u_{\omega, t_{0}}(T)-U(T)\right\|_{H^{1}(\mathbb{R})} \stackrel{|\omega| \rightarrow \infty}{\rightarrow} 0 .
$$

With the same argument as in (3.53), we have

$$
\left\|I_{2}\right\|_{X_{t}} \leq C A\left\|u_{\omega, t_{0}}\right\|_{L_{x}^{5} L_{(T, \infty)}^{10}}^{4}\left\|u_{\omega, t_{0}}\right\|_{X_{(T, \infty)}},
$$

From (4.18), with the use of (4.14) and (4.15), we have

$$
\left\|I_{2}\right\|_{X_{t}} \leq C A(2 \epsilon)^{4} B M_{1}
$$

As in $I_{2}$, using (4.9) and (4.11), we get

$$
\begin{aligned}
\left\|I_{3}\right\|_{X_{t}} & \leq C A\|U\|_{L_{x}^{5} L_{(T, \infty)}^{10}}^{4}\|U\|_{X_{(T, \infty)}} \\
& \leq C A\left(\frac{\epsilon}{4}\right)^{4} B M_{0} .
\end{aligned}
$$

Now given $\beta>0$, we choose $\epsilon>0$ sufficiently small ( $T$ sufficiently large) such that $C A(2 \epsilon)^{4}\left[B M_{0}+B M_{1}\right]<\beta / 3$ and $|\omega|$ sufficiently large, so that (4.16), (4.17), (4.19) and (4.20) imply

$$
\begin{aligned}
\left\|u_{\omega, t_{0}}(t)-U(t)\right\|_{X_{(T, \infty)}} & =\left\|u_{\omega, t_{0}}(T+t)-U(T+t)\right\|_{X_{t}} \\
& \leq\left\|I_{1}\right\|_{X_{t}}+\left\|I_{2}\right\|_{X_{t}}+\left\|I_{3}\right\|_{X_{t}} \\
& <\beta .
\end{aligned}
$$

On the other hand, from Theorem 1.5, we have

$$
\left\|u_{\omega, t_{0}}(t)-U(t)\right\|_{X_{(0, T)}}=\left\|u_{\omega, t_{0}}(t)-U(t)\right\|_{X_{T}} \stackrel{|\omega| \rightarrow \infty}{\longrightarrow} 0 .
$$

Therefore, from (4.21) and (4.22), we can conclude the proof of the theorem. 


\section{References}

[1] Abdullaev, F.K., Caputo, J.G., Kraenkel, R.A., Malomed, B.A.: Controlling collapse in Bose-Einstein condensates by temporal modulation of the scattering length. Phys. Rev. A 67, 012605 (2003)

[2] Bona, J.L., Dougalis, V.A., Karakashian, O.A., McKinney, W.R.: Numerical simulation of singular solutions of the generalized Korteweg-de Vries equation. Contemp. Math. 200, 17-29 (1996)

[3] Bona, J.L., Dougalis, V.A., Karakashian, O.A., McKinney, W.R.: Conservative, high-order numerical schemes for the generalized Korteweg-de Vries equation. Philos. Trans. Roy. Soc. London Ser. A 351, 107-164 (1995)

[4] Bona, J.L., Souganidis, P., Strauss, W.: Stability and instability of solitary waves of Korteweg-de Vries type equation. Proc. Roy. Soc. London Ser. A 411, 395$412(1987)$

[5] Carvajal, X., Panthee, M., Scialom, M.: On the critical KdV equation with timeoscillating nonlinearity. Differ. Integral Equ. 24(5-6), 541-567 (2011)

[6] Cazenave, T., Scialom, M.: A Schrödinger equation with time-oscillating nonlinearity. Revista Matemática Complutense 23, 321-339 (2010)

[7] Damergi, I., Goubet, O.: Blow-up solutions to the nonlinear Schrödinger equation with oscillating nonlinearities. J. Math. Anal. Appl. 352, 336-344 (2009)

[8] Farah, L.G., Linares, F., Pastor, A.: The supercritical generalized KdV equation: global well-posedness in the energy space and below. Math. Res. Lett. 18, 357377 (2011)

[9] Grillakis, M., Shatah, J., Strauss, W.: Stability theory of solitary waves in the presence of symmetry I. J. Funct. Anal. 74, 160-197 (1987)

[10] Kato, T.: On the Cauchy problem for the (generalized) Korteweg-de Vries equation. Adv. Math. Suppl. Stud., Stud. Appl. Math. 8, 93-128 (1983)

[11] Kenig, C.E., Ponce, G., Vega, L.: On the (generalized) Korteweg-de Vries equation. Duke Math. J. 59, 585-610 (1989)

[12] Kenig, C.E., Ponce, G., Vega, L.: Well-posedness of the initial value problem for the Korteweg-de Vries equation. J. Am. Math. Soc. 4(2), 323-347 (1991)

[13] Kenig, C.E., Ponce, G., Vega, L.: Oscillatory integrals and regularity of dispersive equations. Indiana Univ. Math. J 40(1), 33-69 (1991)

[14] Kenig, C.E., Ponce, G., Vega, L.: Well-posedness and scattering results for the generalized Korteweg-de Vries equation via the contraction principle. Comm. Pure Appl. Math. 46, 527-620 (1993)

[15] Kenig, C.E., Ponce, G., Vega, L.: On the concentration of blow up solutions for the generalized $\mathrm{KdV}$ equation critical in $L^{2}$. Nonlinear wave equations (Providence, RI, 1998), pp. 131-156, Contemp. Math., 263. Am. Math. Soc., Providence, RI, 2000 
[16] Kenig, C.E., Ruiz, A.: A strong type $(2,2)$ estimate for a maximal operator associated to the Schrödinger equation. Trans. Am. Math. Soc 230, 239-246 (1983)

[17] Knickerbocker, C.J., Newell, A.C.: Internal solitary waves near a turning point. Phys. Lett. 75(A), 326-330 (1980)

[18] Konotop, V.V., Pacciani, P.: Collapse of solutions of the nonlinear Schrödinger equation with a time dependent nonlinearity: application to the Bose-Einstein condensates. Phys. Rev. Lett. 94, 240405 (2005)

[19] Martel, Y., Merle, F.: Blow up in finite time and dynamics of blow up solutions for the L2-critical generalized KdV equation. J. Amer. Math. Soc. 15, 617$664(2002)$

[20] Martel, Y., Merle, F.: Stability of blow-up profile and lower bounds on blow-up rate for the critical generalized KdV equation. Ann. Math. 155, 235-280 (2002)

[21] Martel, Y., Merle, F.: Instability of solitons for the critical generalized Korteweg-de Vries equation. Geom. Funct. Anal. 11, 74-123 (2001)

[22] Martel, Y., Merle, F., Raphael, P.: Blow up for the critical gKdV equation III: exotic regimes. arXiv:1209.2510

[23] Nunes, W.V.L.: Global well-posedness for the transitional Korteweg-de Vries equation. Appl. Math. Lett. 11(5), 15-20 (1998)

[24] Nunes, W.V.L.: On the well-posedness and scattering for the transitional Benjamin-Ono equation. Mat. Contemp. 3, 127-148 (1992)

M. Panthee

Centro de Matemática

Universidade do Minho

4710-057 Braga

Portugal

e-mail: mpanthee@ime.unicamp.br

M. Panthee and M. Scialom

IMECC-UNICAMP

13083-859 Campinas

São Paulo

Brazil

e-mail: scialom@ime.unicamp.br

Received: 9 February 2012.

Accepted: 21 October 2012. 